ARTICLE

\title{
Synthesized soliton crystals
}

\author{
Zhizhou Lu' ${ }^{1,9}$, Hao-Jing Chen (1) 2,9, Weiqiang Wang ${ }^{1,3,9}$, Lu Yao ${ }^{2,9}$, Yang Wang ${ }^{1,3}$, Yan Yu ${ }^{2}$, B. E. Little ${ }^{1,3}$,

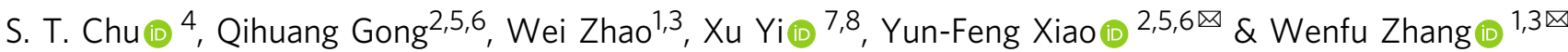

Dissipative Kerr soliton (DKS) featuring broadband coherent frequency comb with compact size and low power consumption, provides an unparalleled tool for nonlinear physics investigation and precise measurement applications. However, the complex nonlinear dynamics generally leads to stochastic soliton formation process and makes it highly challenging to manipulate soliton number and temporal distribution in the microcavity. Here, synthesized and reconfigurable soliton crystals (SCs) are demonstrated by constructing a periodic intra-cavity potential field, which allows deterministic SCs synthesis with soliton numbers from 1 to 32 in a monolithic integrated microcavity. The ordered temporal distribution coherently enhanced the soliton crystal comb lines power up to 3 orders of magnitude in comparison to the single-soliton state. The interaction between the traveling potential field and the soliton crystals creates periodic forces on soliton and results in forced soliton oscillation. Our work paves the way to effectively manipulate cavity solitons. The demonstrated synthesized SCs offer reconfigurable temporal and spectral profiles, which provide compelling advantages for practical applications such as photonic radar, satellite communication and radio-frequency filter.

\footnotetext{
${ }^{1}$ State Key Laboratory of Transient Optics and Photonics, Xi'an Institute of Optics and Precision Mechanics, Chinese Academy of Sciences, Xi'an, China.

${ }^{2}$ State Key Laboratory for Mesoscopic Physics and Frontiers Science Center for Nano-optoelectronics, School of Physics, Peking University, Beijing, China.

${ }^{3}$ University of Chinese Academy of Sciences, Beijing, China. ${ }^{4}$ Department of Physics and Materials Science, City University of Hong Kong, Kowloon Tong, Hong Kong. ${ }^{5}$ Collaborative Innovation Center of Quantum Matter, Beijing, China. ${ }^{6}$ Collaborative Innovation Center of Extreme Optics, Shanxi University, Taiyuan, China. ${ }^{7}$ Department of Electrical and Computer Engineering, University of Virginia, Charlottesville, VA, USA. ${ }^{8}$ Department of Physics, University of Virginia, Charlottesville, VA, USA. ${ }^{9}$ These authors contributed equally: Zhizhou Lu, Hao-Jing Chen, Weiqiang Wang, Lu Yao.

凶email: yfxiao@pku.edu.cn; wfuzhang@opt.ac.cn
} 
D issipative Kerr solitons (DKSs) are self-organized wave packets in photonic-chip-based microcavities ${ }^{1-7}$, where the chromatic dispersion is balanced by Kerr nonlinear shift, and the cavity dissipation is offset by Kerr parametric gain. DKSs produce ultrashort pulse trains and equally spaced comb lines $^{8-15}$, and have attracted significant interest for applications, including optical communications ${ }^{16,17}$, spectroscopy ${ }^{18,19}$, ultrafast ranging ${ }^{20-23}$, low-noise microwave generation ${ }^{24}$, frequency synthesis $^{25} /$ division $^{26}$, and quantum key distribution $(\mathrm{QKD})^{27}$, etc. Up to date, various soliton states have been theoretically and experimentally demonstrated, such as Stokes solitons ${ }^{28}$, BrillouinKerr solitons ${ }^{29}$, breather solitons $s^{30-34}$, laser cavity solitons ${ }^{35}$ as well as soliton crystals (SC) ${ }^{36-39}$. Particularly, perfect soliton crystals (PSC) ${ }^{38,39}$, where temporal solitons are equally spaced in the microresonator, are of great interest. While preserving many characteristics from the single-soliton state, i.e., smooth spectral envelope and highly ordered temporal soliton distribution, they offer higher comb power per line and flexible soliton repetition rate, which are critical for microcomb applications. Previously, the generation and stabilization of SC rely on avoided mode crossing (AMX) induced background potential field ${ }^{36,38}$ or

a

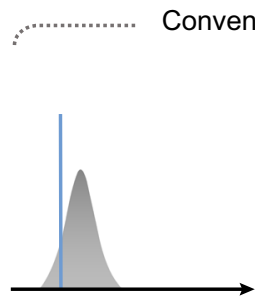

Frequency
C

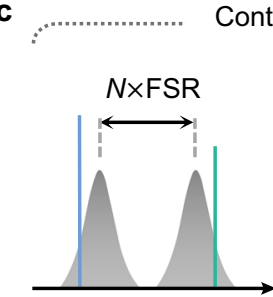

Frequency

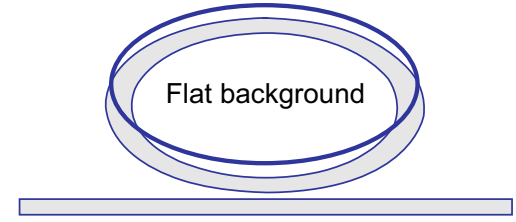

rollable dichromatic pumping .....................

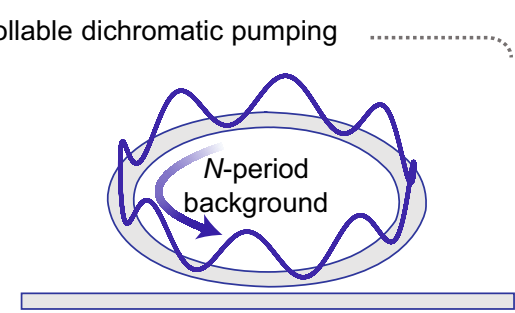

e

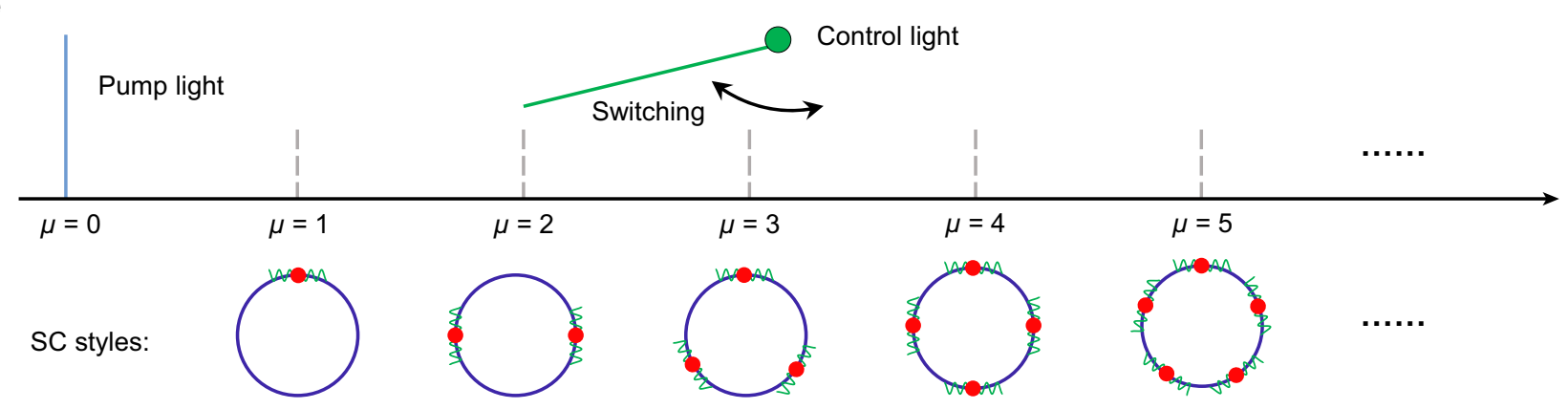

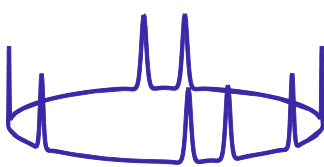

Field envelope

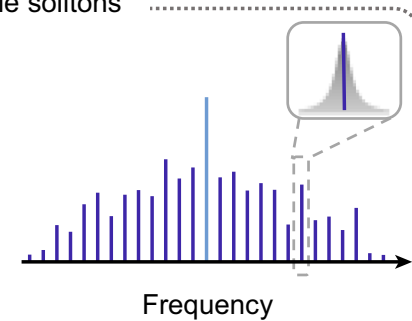

d

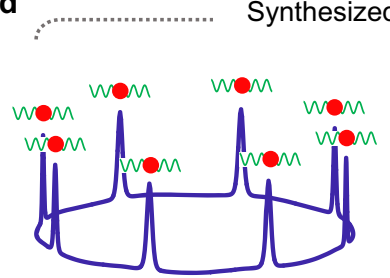

Field envelope
Frequency

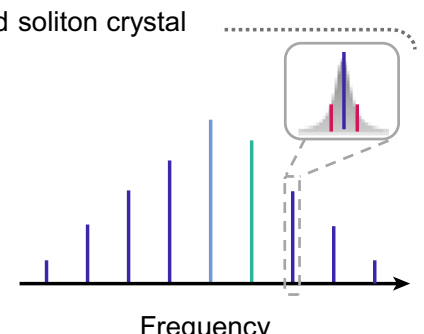

delicate control of the pump condition ${ }^{39}$. However, the above schemes both have complex dynamics processes and require extreme fabrication precision to reproduce or demand materialspecific properties, which cannot offer a wide-range, universal switching for SC states.

In this article, we propose and demonstrate on-demand synthesis and manipulation of a library of PSCs through a controlled potential field. The $N$-period modulated background potential field is constructed by pumping the cavity with an additional control light at the frequency which is $\mathrm{N}$-mode spaced from the primary pump light. As the period and intensity of the potential field can be conveniently tuned by the control light, our method offers full reconfigurable capability to the PSCs generation. We demonstrate synthesized SCs with a given soliton number $N$ from 1 to 32 on demand, equivalent to a reconfigurable soliton generator with a repetition rate ranging from $\sim 49 \mathrm{GHz}$ to $\sim 1.57 \mathrm{THz}$. Furthermore, the group velocity mismatch between the SC and potential field ${ }^{40,41}$ are investigated both theoretically and experimentally, which induces forced oscillation in the power and intracavity position of the synthesized $\mathrm{SCs}^{42}$. Finally, we show that the repetition rate of the synthesized SC changes

b

Multiple solitons 
a

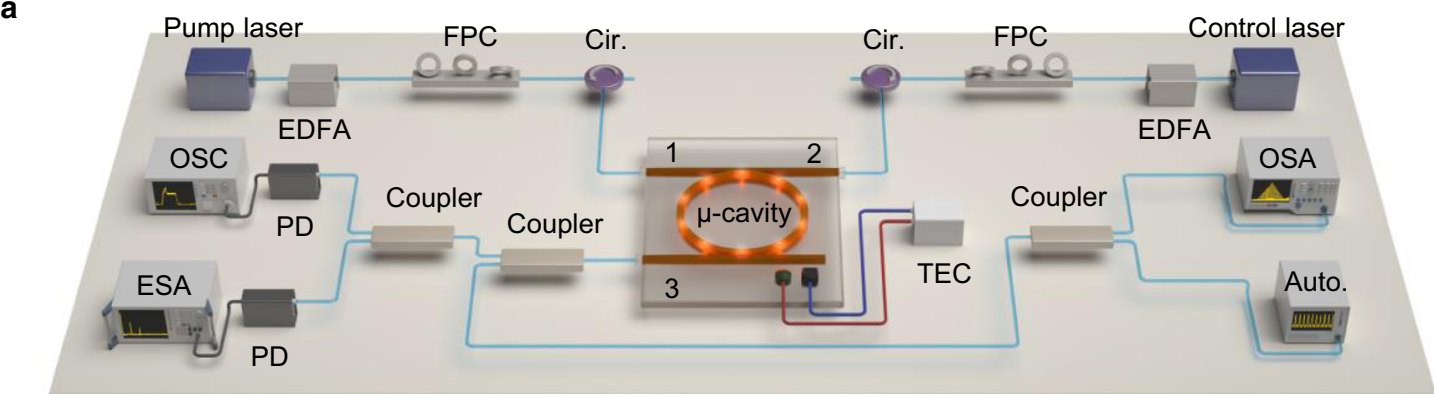

b

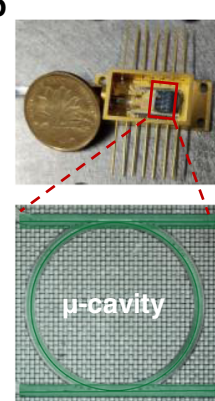

C

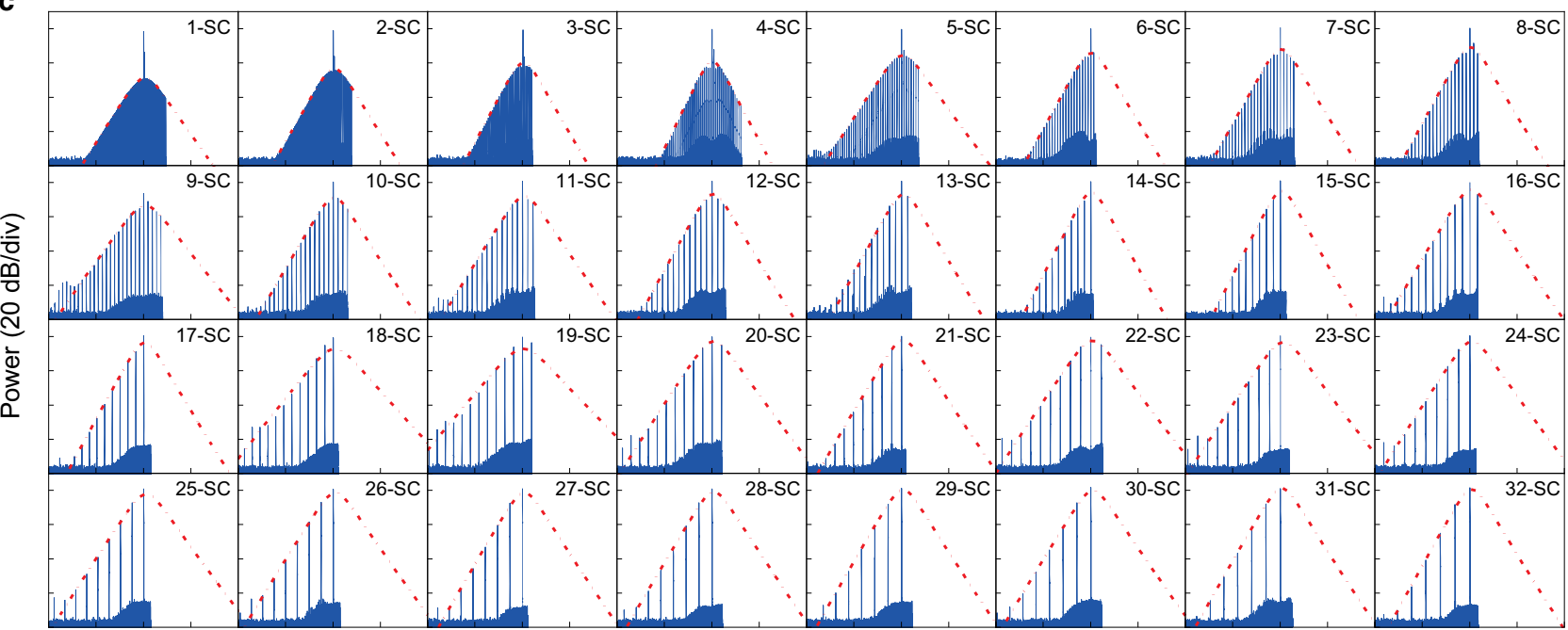

Wavelength $(40 \mathrm{~nm} / \mathrm{div})$

Fig. 2 A library of 1-32 soliton crystals. a Illustration of the experimental set-up, the wavelength of the pump light is fixed, while the wavelength of the control light is tunable for both SC switching and intracavity thermal balancing. EDFA erbium-doped fiber amplifier, FPC fiber polarization controller, Cir. circulator, PD photodiode, TEC thermoelectric cooler, OSC oscilloscope, ESA electric spectrum analyzer, OSA optical spectrum analyzer, Auto.

autocorrelator. b Butterfly-packaged device with a 20.5-mm-diameter Chinese coin for comparison (upper panel). Microscope image of the high-index doped silica glass microring resonators with a diameter of $\sim 1.2 \mathrm{~mm}$ (lower panel). c Complete optical spectra for 1-32 synthesized SC with smooth sech ${ }^{2}$ envelope (red dashed line).

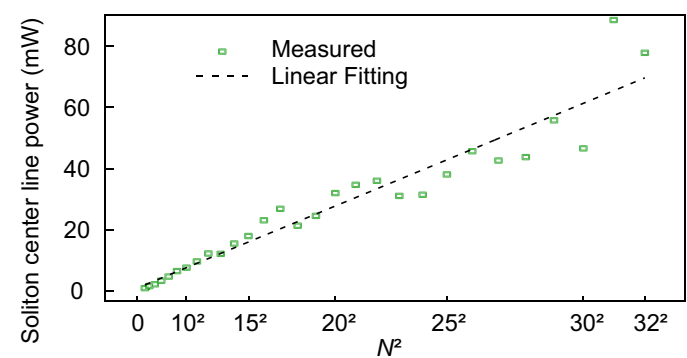

Fig. 3 Enhancement of soliton comb line power. The soliton center line power extracted from experimental spetral envolopes versus square of soliton number $N^{2}$, which shows $N^{2}$ enhancement in comparison to the single-soliton state excited under similar pump conditions.

linearly with the relative velocity between the potential field and the SC. The mechanism shown in this work can be extended to other platforms to greatly enhance the flexibility of on-chip SC for practical applications.

\section{Results}

Synthesized SCs. In a conventional monochromatic pumping scheme, the cavity soliton is initialized from a flat CW background field (Fig. 1a). The uniformly distributed gain makes the positions and quantity of the generated solitons unpredictable ${ }^{43}$, resulting in irregular multiple soliton pulse train and unsmooth spectral envelope (Fig. 1b). In our approach, a second CW laser is introduced as a control light to provide a periodically modulated $\mathrm{CW}$ background from the two-beam beating and control the SC generation process. The periodic number of potential field $N$ is equal to the mode number spacing between the primary pump light and control light. Figure 2a shows the experimental setup for SC synthesis. The pump laser and the control laser are amplified and coupled to a single high- $Q$ microcavity resonance from two counter-propagating directions via two fiber circulators. The role of the control laser is twofold. On the one hand, a small portion of the control light is back-scattered to the opposite direction and beats with the co-propagating clockwise pump light, constructing a periodically modulated background field. On the other hand, the main part of the control light propagating in the counterclockwise direction is adopted to stabilize the cavity temperature when the pump light transitions into the red-detuned regime ${ }^{40,44}$. The add-drop type microcavity is fabricated on a CMOScompatible high-index doped silica glass platform ${ }^{45,46}$. The $Q$ factor and free spectral range (FSR) of the microresonator are $\sim 2.63$ million and $\sim 48.9 \mathrm{GHz}$, respectively. To couple the light onto the chip, a standard $250 \mu \mathrm{m}$ pitch fiber array glued to the on-chip bus waveguide is used 47,48 . The microcavity is butterflypackaged with a thermo-electric cooler (TEC) which is controlled by an external controller ${ }^{12,37}$ 
a

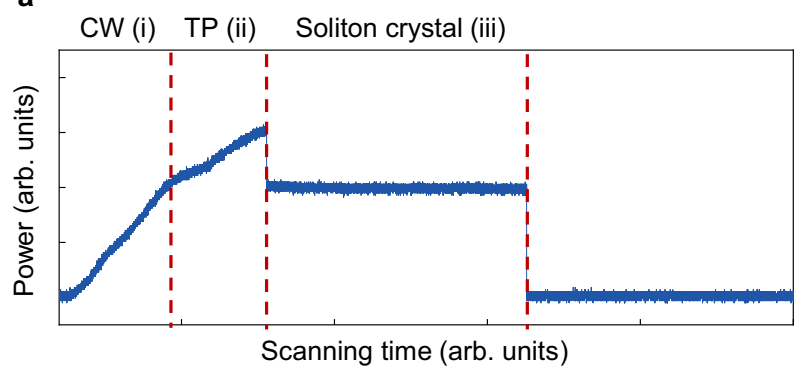

C

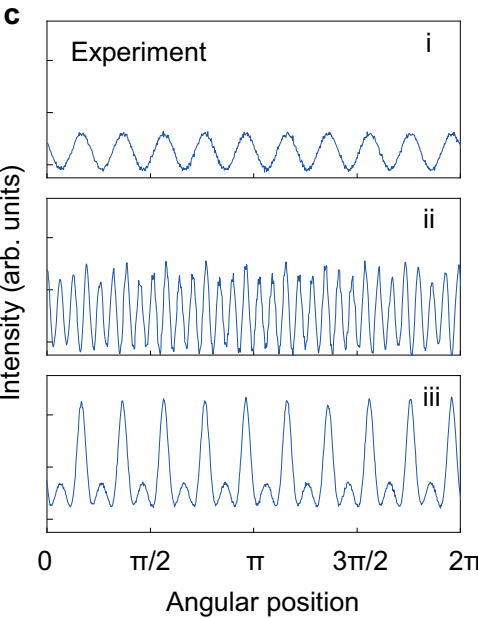

d

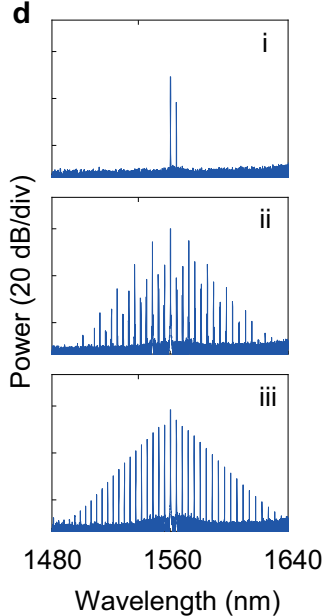

b

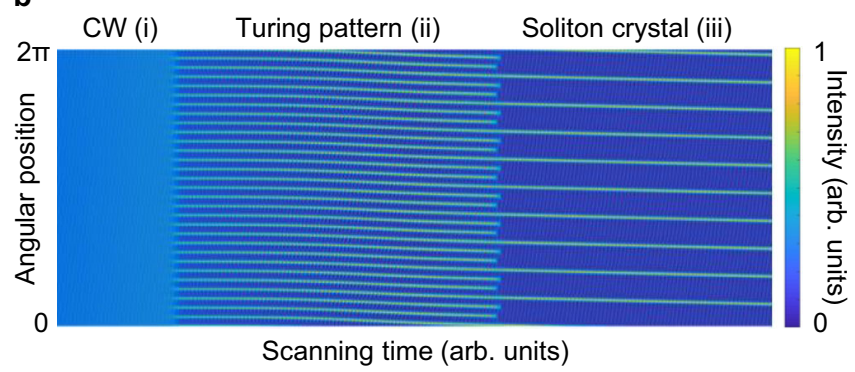

e

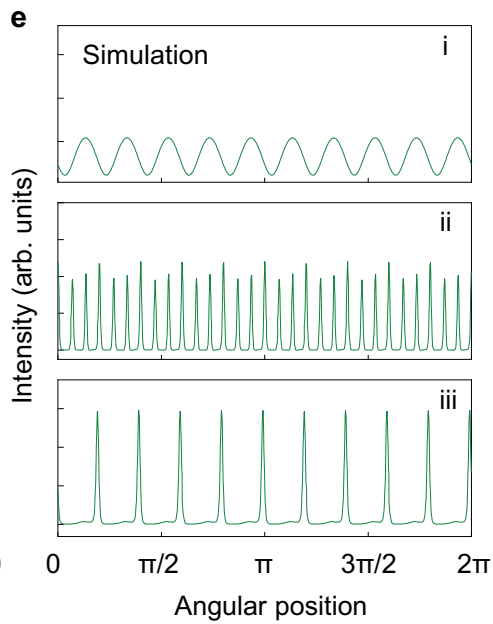

f

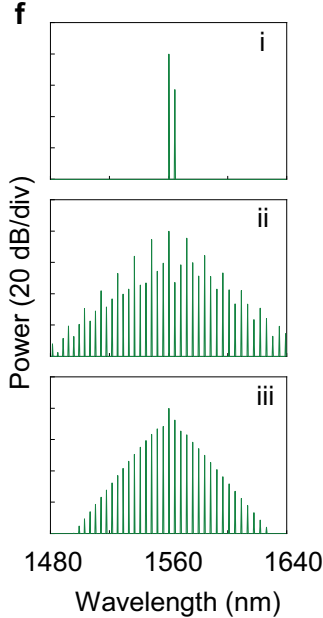

Fig. 4 Formation dynamics of soliton crystal. a Measured intracavity power evolution trace. With cavity resonance scanning from longer wavelength to shorter wavelength gradually, the laser-cavity detuning varies from blue detuned to red-detuned regime. Three states are respectively marked as: state i, CW background; state ii, modulated Turing pattern (TP); state iii, soliton crystal (SC). The deterministic "step" in the trace is a characteristic feature of SC formation. $\mathbf{b}$ Simulations of the intracavity temporal waveform evolution. Turing combs directly turns to SC state as the pump enters red-detuned regime. c, d Measured temporal (c) and spectral (d) profile in different states: modulated CW background (i), modulated TP (ii), SC (iii). e, f Simulated angular (e) and spectral (f) profile in different states, showing good agreement with the experiment.

In the experiment, SC can be generated reliably by slowly decreasing the operating temperature through the TEC controller to sweep cavity resonance from blue-detuned to the red-detuned regime. The temporal and spectral profiles are measured using an autocorrelator and an optical spectrum analyzer (OSA), respectively. By switching the mode number spacing between the control and pump light, a library of $1-32$ SC with repetition rates from $1 \times$ to $32 \times$ FSR of the microcavity, are deterministically realized with high repeatability (see section 3.1 of SI for more experimental evidence). As shown in Fig. 2c, the measured spectra are all smooth with a $\operatorname{sech}^{2}$ envelope and the comb line power is enhanced by $N^{2}$ (Fig. 3), thanks to the ordered distribution of the synthesized $\mathrm{SC}^{38}$. Moreover, the spatial overlap between the CW pump light and the soliton pulses becomes larger with the increasing soliton number, providing great potential to boost the soliton conversion efficiency. The demonstrated reconfigurable and high-power SC in a monolithic microcavity provides an ideal toolroom for tunable microwave/ millimeter-wave photonics ${ }^{49,50}$.

We then focus on understanding the formation dynamics of the SC by taking 10-SC as an example. The wavelength of the pump and control light are 1560.2 and $1564.1986 \mathrm{~nm}(\sim 10$ FSR away from pump), respectively. The evolution of the power is recorded while the temperature of the microcavity is gradually decreased to scan the pump-cavity resonances from longer wavelength to shorter wavelength side (Fig. 4a). Three typical stages are clearly recognized: (i) CW, (ii) modulated Turing pattern (TP) and (iii) SC. These stages are characterized by simultaneously measuring the intracavity optical field in temporal
(Fig. 4c) and spectral (Fig. 4d) domain. The SC formation is recognized by the characteristic "step" power trace, ordered temporal pulse distribution, as well as the smooth sech ${ }^{2}$ spectral envelope. The soliton formation is able to bypass the chaotic regime, and is directly evolved from the modulated TP stage. The modulation field causes the multiple Turing pulses turn into $N$ equally spaced solitons, i.e., N-SC. The evolution process of sythesized SC is well reproduced by numerical simulation of dichromatic-pump Lugiato-Lefever equation (DLLE, see SI for details). When the pump detuning is gradually increased, the three stages discussed above are sequentially distinguished, as shown in the simulated power trace in the temporal evolution trace in Fig. 4b. The simulated temporal waveforms and spectra of the three representative stages are shown in Fig. 4e, $\mathrm{f}$, which agree well with the experiment.

Forced oscillation of SCs. In the course of generating synthesized SCs, a novel type of soliton oscillation is observed when the modulated CW background field has a relative velocity to SC. This relative motion of the modulated background field creates an moving offset (phase difference) between the potential field and the SC, which provides a periodic force on the soliton ${ }^{51}$. Such force is derived as (See SI for details):

$$
F=F_{0} \cos \left(\eta \phi_{c}-\Delta \widetilde{\omega} \tau\right)
$$

Here $F_{0}$ is the amplitude of $F$, which is proportional to the amplitude of control light. $\eta=\mu_{c}-\mu_{p}$ is the mode number spacing between the pump light and control light. $\phi_{c}$ is the angular position of soliton center, $\tau$ is the normalized slow time. 
a

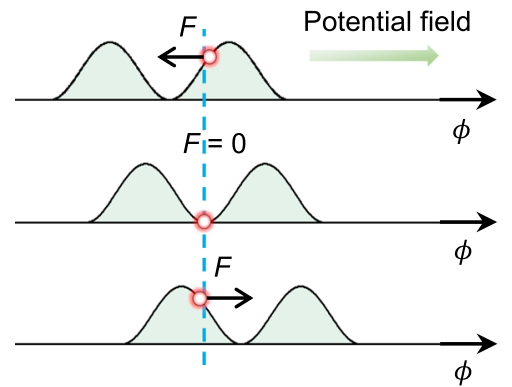

d

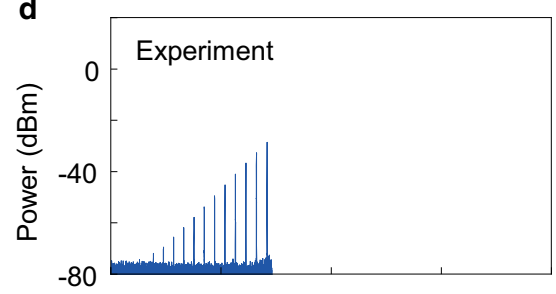

e

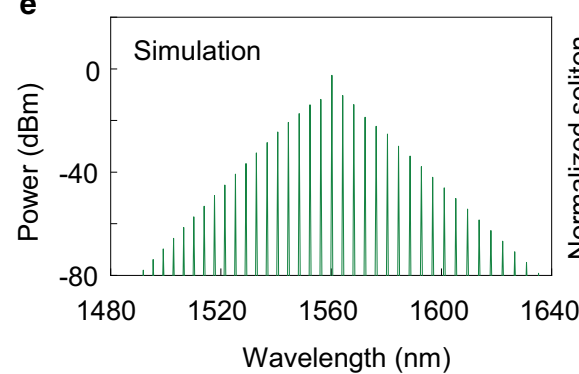

b

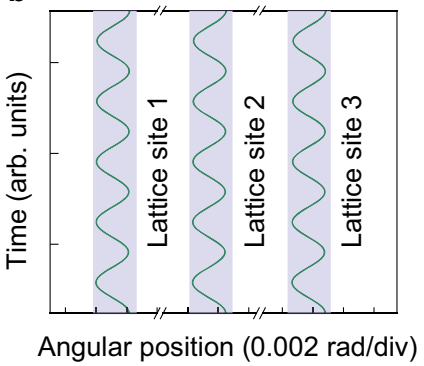

c

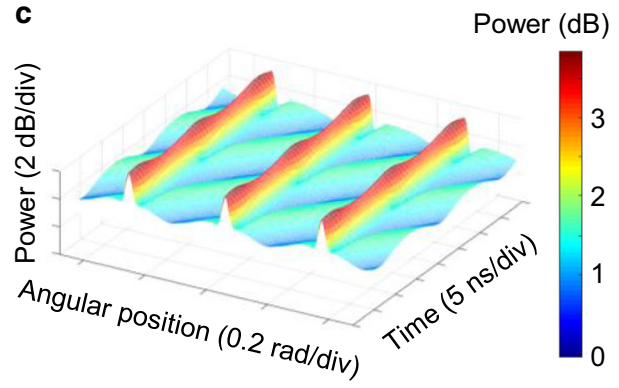

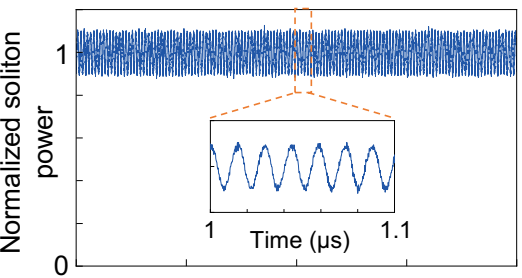
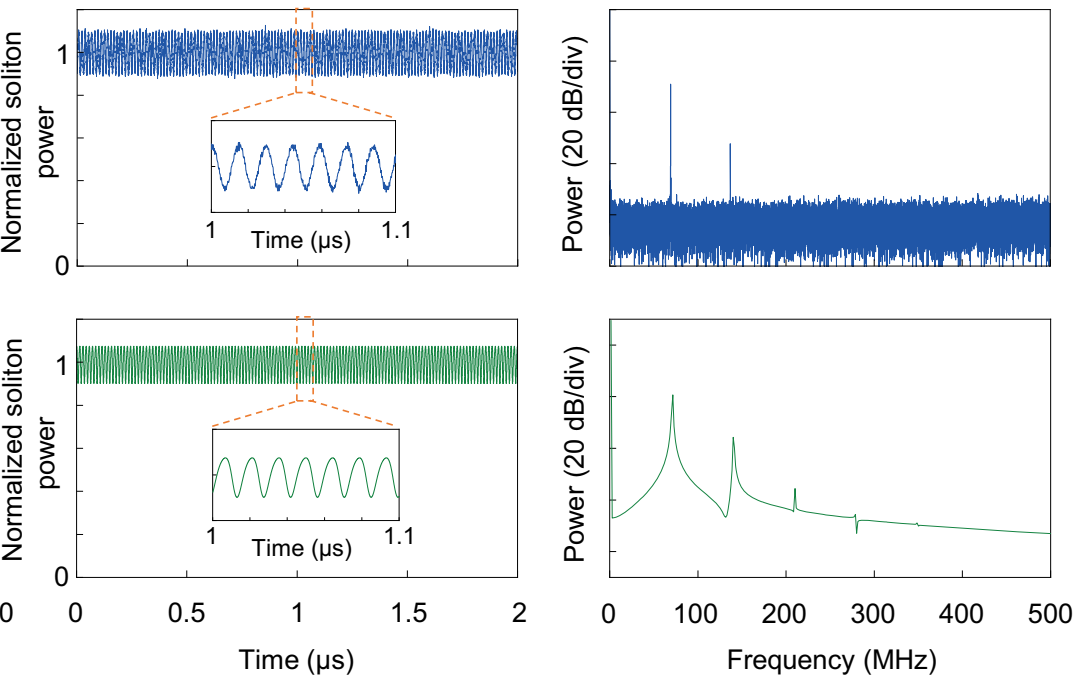

Fig. 5 Forced oscillation of SC. a Illustration showing force applied on SC. The moving potential field imposes a periodic force on soliton pulses, resulting in the forced oscillatory motion of SC. b Simulated intracavity angular position oscillation of 3 lattice sites from a 10-SC state. Simulated intracavity field envelope is shown in (c). The modulated background has a relative moving speed to SC, and SC vibrates around its equilibrium position with oscillating power. d, e Experimental (d) and simulated (e) optical spectrum (left panel), normalized soliton power trace (middle panel, inset: zoom-in of 1-1.1 4 s range) and electrical spectrum (right panel) for 10-SC state. Electrical spectra show that the fundamental oscillation frequency is $69 \mathrm{MHz}$.

$\Delta \widetilde{\omega}=2\left(\omega_{c}-\omega_{p}-\eta D_{1}\right) / \kappa$ is the normalized mismatch angular frequency. As a result, the SCs oscillate around their equilibrium positions periodically (Fig. 5a). To gain more insights into the intracavity dynamics, we first define the soliton momentum as (see SI for details): $P=2 \beta \sum_{\mu} \mu\left|\psi_{\mu}\right|^{2}$, here $2 \beta \mu$ and $\left|\psi_{\mu}\right|^{2}$ represent the normalized relative group velocity and photon number of mode $\mu$, respectively. Based on the momentum analysis, the dynamical equation for SCs can be derived:

$$
\frac{\mathrm{d}^{2} \phi_{c}}{\mathrm{~d} \tau^{2}}+2 \frac{\mathrm{d} \phi_{c}}{\mathrm{~d} \tau}-\frac{F}{M}=0
$$

The above equation is a typical forced oscillation equation. The effective kinetic mass of soliton is derived as $M=E$ (given by momentum analysis, see SI for details), where $E$ is the optical pulse energy. Based on Eq. (2), we calculate the evolution trace of the SC angular position. As shown in Fig. 5b, where three adjacent lattice sites of a 10-SC oscillate in phase. The amplitude of the oscillation is calculated as $\sim 0.001 \mathrm{rad}$ ( $\sim 4 \%$ of the soliton width).

The forced oscillation also induces the periodical variation of $\mathrm{CW}$, and causes the SC power to oscillate. The measured normalized soliton power trace shows the periodic modulation (middle panel of Fig. 5 d), with a modulation depth of $\sim 10 \%$. The modulation frequency is $69 \mathrm{MHz}$, which is verified by electrical measurement, see right panel of Fig. $5 \mathrm{~d}$, where the second harmonic of $138 \mathrm{MHz}$ is also detected. It should be noted that this power oscillation is fundamentally distinct from the power breathing in the breather soliton, as its optical spectrum maintains the stable $\operatorname{sech}^{2}$ envelope instead of the typical triangle envelope of breather ${ }^{31,32}$. These experimental observations are further validated by DLLE-based numerical simulations (Fig. 5e). The forced oscillation corresponds to sidebands generation around the comb line in the forms of cross-phase modulation (XPM) and subsequent FWM comb lines caused by XPM and SC comb, the experimental and numerical characterization of XPM comb are included in SI. The forced oscillation of the SC may be eliminated once the control beam is switched off. However, the process of switching off the control beam while maintaining the soliton states is complicated by the thermal effect in the microresonator. Further investigation to reduce the thermal effect is required to eliminate the control beam after the formation of SC states, and this is possible through optimizing the fabrication process of microresonators ${ }^{52,53}$.

Soliton oscillation frequency and repetition rate tuning. Furthermore, due to the photothermal effect, the oscillation frequency and the repetition rate of synthesized SC can be tuned by adjusting the frequency of the control light. As the single-soliton state has an electronic-detectable repetition rate, we study the tuning process of a single-soliton state as an example. Experimentally, the oscillation frequency and repetition rate are directly measured using two high-speed ESAs to monitor the tuning process. The frequency of the control laser is tuned finely by 

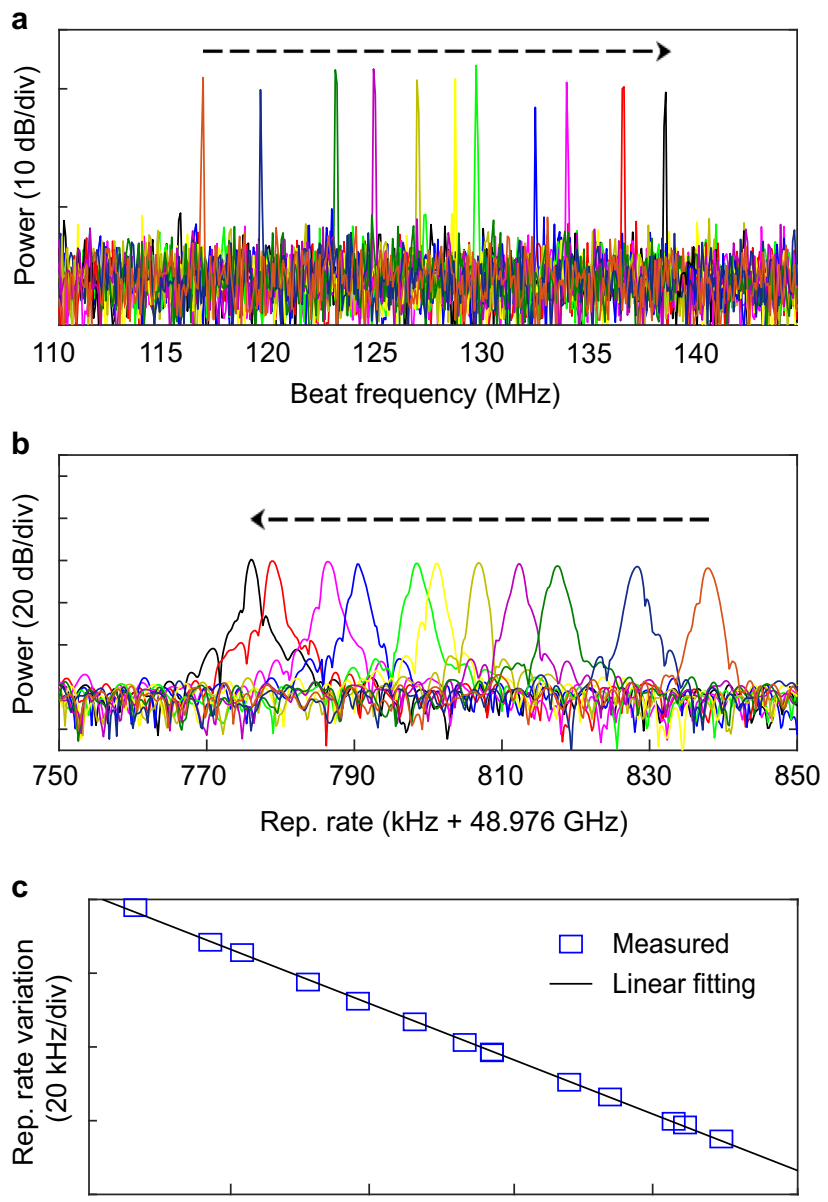

Beat frequency variation ( $5 \mathrm{MHz} / \mathrm{div})$

Fig. 6 Soliton repetition rate tuning. $\mathbf{a}, \mathbf{b}$ The measured beat frequency $\Delta f$ (i.e., soliton oscillation frequency) and repetition rate of a single soliton as the frequency of the control laser is tuned. c The repetition rate of the single soliton is approximately linearly decreasing with the increasing beat frequency, providing a way for realizing oscillation frequency and repetition rate tuning.

gradually changing the driven current of ECDL. For each driven current of the control light, we record both the oscillation frequency and the repetition rate of the single-soliton comb, as shown in Fig. 6a, b, respectively.

In our measurement, the oscillation frequency of the soliton microcomb is tuned over $20.6 \mathrm{MHz}$ (Fig. 6a), and the repetition rate changes about $60 \mathrm{kHz}$ (Fig. 6b). The soliton repetition rate is linearly changed along with the oscillation frequency (Fig. 6c). It provides a way to finely tune soliton repetition rate and oscillation frequency.

\section{Discussion}

Here, we explore the SC synthesis on demand and the forced oscillation process of the SC. The artificial background potential field is potentially reconfigurable in real-time, provides a platform for studying the cavity Kerr soliton dynamics. The demonstrated reconfigurable SC microcombs in a monolithic integrated microresontors with the repetition rate covering broad microwave bands (including $\mathrm{V}$ band, $\mathrm{W}$ band, and $\mathrm{G}$ band) and $\mathrm{THz}$ band, has great potential in applications such as satellite communications ${ }^{54}$, photonic $\operatorname{radar}^{55}$, radio-frequency filter ${ }^{56,57}$, and $\mathrm{THz}$ technologies. The proposed approach is easy to implement, and therefore applicable to most materials and different cavity configurations.

\section{Methods}

Controllable artificial background field. A pre-designed background is proposed to change the soliton generation dynamics and regulate the soliton distribution and quantity. Operationally, this can be realized by adding an additional control light that is $N \times$ FSR from the primary pump light. This leads to the formation of controllable artificial background field expressed as:

$\widetilde{E_{b}}=E_{p} e^{-i \omega_{p} t} e^{i \mu_{p} \phi_{l}}+E_{c} e^{-i \omega_{c} t} e^{i \mu_{c} \phi_{l}}$, where $E_{p, c}$ and $\omega_{p, c}$ represent the electric field amplitude and angular frequency of the pump (control) laser, respectively. $\phi_{1}$ is the polar angle in lab coordinate. In the rotating angular coordinate $\phi=\phi_{l}-D_{1} t$ where $D_{1}=2 \pi \times F S R$, the background field can be rewritten as $\widetilde{E_{b}}=\left(E_{p}+E_{c} e^{-i \Delta \omega t} e^{i \eta \phi}\right) e^{-i \omega_{p} t} e^{i \mu_{p} \phi_{l}}$, which is $N$-periodically modulated $(N=\eta=$ $\left.\mu_{c}-\mu_{p}\right) . \Delta \omega=\omega_{c}-\omega_{p}-\eta D_{1}$ is the frequency mismatch. $\Delta \omega / \eta$ is the traveling speed of the background field, which could be adjusted by tuning the frequency (detuning) of the control light (detailed derivation see Supplementary information). The modulated background field redistributes the refractive index of the cavity in a periodic fashion (Fig. 1c), and regularizes the soliton formation probability around the cavity. Figure 1d shows a synthesized SC where the solitons are evenly distributed with a smooth spectral envelope. Note that if there is a relative motion between the SC and the field, i.e., $\Delta \omega$ is detuned from zero, the SC would periodically oscillate in the temporal domain, which corresponds to modulation sidebands generation in the frequency domain as shown in the inset of Fig. 1d.

\section{Data availability}

The data that support the findings of this study are available from the corresponding author upon reasonable request.

\section{Code availability}

The code for the data analysis in this article is available from the corresponding author upon reasonable request.

Received: 20 November 2020; Accepted: 16 April 2021;

Published online: 26 May 2021

\section{References}

1. Kippenberg, T. J., Gaeta, A. L., Lipson, M. \& Gorodetsky, M. L. Dissipative Kerr solitons in optical microresonators. Science 361, eaan8083 (2018).

2. Tanabe, T., Fujii, S. \& Suzuki, R. Review on microresonator frequency combs Jpn. J. Appl. Phys. 58, SJ0801 (2019).

3. Gaeta, A. L., Lipson, M. \& Kippenberg, T. J. Photonic-chip-based frequency combs. Nat. Photon. 13, 158-169 (2019).

4. Chembo, Y. K. Kerr optical frequency combs: theory, applications and perspectives. Nanophotonics 5, 214-230 (2016).

5. Diddams, S. A., Vahala, K. \& Udem, T. Optical frequency combs: coherently uniting the electromagnetic spectrum. Science 369, 267-267 (2020).

6. Wang, W., Wang, L. \& Zhang, W. Advances in soliton microcomb generation. Adv. Photonics 2, 34001 (2020).

7. Pasquazi, A., Peccianti, M., Razzari, L., Moss, D. J. \& Morandotti, R. Microcombs: a novel generation of optical sources. Phys. Rep. 729, 1-81 (2017).

8. Herr, T. et al. Temporal solitons in optical microresonators. Nat. Photon. 8, 145-152 (2014).

9. Yi, X., Yang, Q.-F., Yang, K. Y., Suh, M.-G. \& Vahala, K. Soliton frequency comb at microwave rates in a high-Q silica microresonator. Optica 2, 1078-1085 (2015).

10. Brasch, V. et al. Photonic chip-based optical frequency comb using soliton Cherenkov radiation. Science 351, 357-360 (2016).

11. Gong, Z. et al. High-fidelity cavity soliton generation in crystalline AlN microring resonators. Opt. Lett. 43, 4366-4369 (2018).

12. Lu, Z. et al. Deterministic generation and switching of dissipative Kerr soliton in a thermally controlled micro-resonator. AIP $A d v$. 9, 25314 (2019).

13. Chen, H.-J. et al. Chaos-assisted two-octave-spanning microcombs. Nat. Commun. 11, 1-6 (2020).

14. Gong, Z. et al. Soliton microcomb generation at $2 \mu \mathrm{m}$ in $\mathrm{z}$-cut lithium niobate microring resonators. Opt. Lett. 44, 3182-3185 (2019).

15. Wang, P.-H. et al. Intracavity characterization of micro-comb generation in the single-soliton regime. Opt. Express 24, 10890-10897 (2016).

16. Marin-Palomo, P. et al. Microresonator-based solitons for massively parallel coherent optical communications. Nature 546, 274-279 (2017).

17. Geng, Y. et al. Terabit optical OFDM superchannel transmission via coherent carriers of a hybrid chip-scale soliton frequency comb. Opt. Lett. 43 2406-2409 (2018) 
18. Suh, M.-G., Yang, Q.-F., Yang, K. Y., Yi, X. \& Vahala, K. J. Microresonator soliton dual-comb spectroscopy. Science 354, 600-603 (2016).

19. Yu, M. et al. Silicon-chip-based mid-infrared dual-comb spectroscopy. Nat. Commun. 9, 1869 (2018).

20. Suh, M.-G. \& Vahala, K. J. Soliton microcomb range measurement. Science 359, 884-887 (2018).

21. Trocha, P. et al. Ultrafast optical ranging using microresonator soliton frequency combs. Science 359, 887-891 (2018).

22. Wang, J. et al. Long-distance ranging with high precision using a soliton microcomb. Photon. Res. 8, 1964-1972 (2020).

23. Jang, Y.-S. et al. Nanometric precision distance metrology via hybrid spectrally resolved and homodyne interferometry in a single soliton frequency microcomb. Phys. Rev. Lett. 126, 23903 (2021).

24. Liang, W. et al. High spectral purity Kerr frequency comb radio frequency photonic oscillator. Nat. Commun. 6, 7957 (2015).

25. Spencer, D. T. et al. An optical-frequency synthesizer using integrated photonics. Nature 557, 81-85 (2018).

26. Wang, B., Yang, Z., Zhang, X. \& Yi, X. Vernier frequency division with dualmicroresonator solitons. Nat. Commun. 11, 3975 (2020).

27. Wang, F. et al. Quantum key distribution with on-chip dissipative Kerr soliton. Laser Photon. Rev. 14, 1900190 (2020).

28. Yang, Q.-F., Yi, X., Yang, K. Y. \& Vahala, K. Stokes solitons in optical microcavities. Nat. Phys. 13, 53-57 (2017).

29. Bai, Y. et al. Brillouin-Kerr soliton frequency combs in an optical microresonator. Phys. Rev. Lett. 126, 063901 (2021).

30. Bao, C. et al. Observation of Fermi-Pasta-Ulam recurrence induced by breather solitons in an optical microresonator. Phys. Rev. Lett. 117, 163901 (2016).

31. $\mathrm{Yu}, \mathrm{M}$. et al. Breather soliton dynamics in microresonators. Nat. Commun. 8 14569 (2017)

32. Lucas, E., Karpov, M., Guo, H., Gorodetsky, M. \& Kippenberg, T. Breathing dissipative solitons in optical microresonators. Nat. Commun. 8, 736 (2017).

33. Guo, H. et al. Intermode breather solitons in optical microresonators. Phys. Rev. X 7, 41055 (2017).

34. Wan, $\mathrm{S}$. et al. Frequency stabilization and tuning of breathing solitons in $\mathrm{Si}_{3} \mathrm{~N}_{4}$ microresonators. Photon. Res. 8, 1342-1349 (2020).

35. Bao, H. et al. Laser cavity-soliton microcombs. Nat. Photon. 13, 384-389 (2019).

36. Cole, D. C., Lamb, E. S., Del'Haye, P., Diddams, S. A. \& Papp, S. B. Soliton crystals in Kerr resonators. Nat. Photon. 11, 671 (2017).

37. Wang, W. et al. Robust soliton crystals in a thermally controlled microresonator. Opt. Lett. 43, 2002-2005 (2018).

38. Karpov, M. et al. Dynamics of soliton crystals in optical microresonators. Nat. Phys. 15, 1071-1077 (2019).

39. He, Y., Ling, J., Li, M. \& Lin, Q. Perfect soliton crystals on demand. Laser Photon. Rev. 14, 1900339 (2020).

40. Zhou, H. et al. Soliton bursts and deterministic dissipative Kerr soliton generation in auxiliary-assisted microcavities. Light Sci. Appl. 8, 50 (2019).

41. Zhang, S., Silver, J. M., Bi, T. \& Del'Haye, P. Spectral extension and synchronization of microcombs in a single microresonator. Nat. Commun. 11, 6384 (2020)

42. Bao, C. et al. Oscillatory motion of a counterpropagating Kerr soliton dimer. Phys. Rev. A 103, L011501 (2021).

43. Guo, H. et al. Universal dynamics and deterministic switching of dissipative Kerr solitons in optical microresonators. Nat. Phys. 13, 94-102 (2017).

44. Zhang, S. Y. et al. Sub-milliwatt-level microresonator solitons with extended access range using an auxiliary laser. Optica 6, 206-212 (2019).

45. Moss, D. J., Morandotti, R., Gaeta, A. L. \& Lipson, M. New CMOS-compatible platforms based on silicon nitride and Hydex for nonlinear optics. Nat. Photon. 7, 597-607 (2013).

46. Wang, W. et al. Repetition rate multiplication pulsed laser source based on a microring resonator. ACS Photon. 4, 1677-1683 (2017).

47. Corcoran, B. et al. Ultra-dense optical data transmission over standard fibre with a single chip source. Nat. Commun. 11, 2568 (2020).

48. Wang, X. et al. Program-controlled single soliton microcomb source. Photon. Res. 9, 66-72 (2021).

49. Roh, W. et al. Millimeter-wave beamforming as an enabling technology for 5G cellular communications: theoretical feasibility and prototype results. IEEE Commun. Mag. 52, 106-113 (2014).

50. Wang, B. et al. Towards high-power, high-coherence, integrated photonic mmwave platform with microcavity solitons. Light Sci. Appl. 10, 1-10 (2021).

51. Taheri, H., Matsko, A. B. \& Maleki, L. Optical lattice trap for Kerr solitons. Eur. Phys. J. D. 71, 1-13 (2017).
52. Liu, J. et al. Ultralow-power chip-based soliton microcombs for photonic integration. Optica 5, 1347-1353 (2018).

53. Izhaky, N. et al. Development of CMOS-compatible integrated silicon photonics devices. IEEE J. Sel. Top. Quant. 12, 1688-1698 (2006).

54. Federici, J. \& Moeller, L. Review of terahertz and subterahertz wireless communications. J. Appl. Phys. 107, 111101 (2010).

55. Riemensberger, J. et al. Massively parallel coherent laser ranging using a soliton microcomb. Nature 581, 164-170 (2020)

56. $\mathrm{Xu}, \mathrm{X}$. et al. Advanced $\mathrm{RF}$ and microwave functions based on an integrated optical frequency comb source. Opt. Express 26, 2569-2583 (2018).

57. $\mathrm{Hu}$, J. et al. Reconfigurable radiofrequency filters based on versatile soliton microcombs. Nat. Commun. 11, 4377 (2020).

\section{Acknowledgements}

The authors thank B. Li, J.-h. Chen, Q.-T. Cao, Q.-X. Ji, Q.-F. Yang, J. Liu, L. R. Wang, W. C. Fan, X. F. Wang, and M. L. Liu for helpful discussions. The authors thank S. P. Dang for providing high-resolution image of the microcavity. This project is supported by the National Key R\&D Program of China (Grant No. 2016YFA0301302 and No. 2018YFB2200401), the National Natural Science Foundation of China (Grant Nos. $11825402,11654003,62075238,12041602,61635013$, and 61675231), Beijing Municipal Science \& Technology Commission No. Z201100004020007, Beijing Academy of Quantum Information Sciences (Y18G20), Key R\&D Program of Guangdong Province (2018B030329001), the Strategic Priority Research Program of the Chinese Academy of Sciences (Grant No. XDB24030600), and the High-performance Computing Platform of Peking University. X.Y. is supported by U.S. National Science Foundation (Award No. 1842641)

\section{Author contributions}

W.Z., Y.-F.X., and X.Y. conceived the idea. Z.L., H.-J.C., Y.W., L.Y., and W.W. built the experimental set-up and performed the measurements. W.W., W.Z., B.E.L., and S.T.C. designed and fabricated the device. W.W. packaged the microcavity. H.-J.C, Y.Y., L.Y., and Z.L. built the theoretical models and performed numerical simulations. All authors analyzed the data, participated in preparing the manuscript, and contributed to the discussions. W.Z., Y.-F.X., W.Z., and Q.G. supervised the project.

\section{Competing interests}

The authors declare no competing interests.

\section{Additional information}

Supplementary information The online version contains supplementary material available at https://doi.org/10.1038/s41467-021-23172-2.

Correspondence and requests for materials should be addressed to Y.-F.X. or W.Z.

Peer review information Nature Communications thanks Curtis Menyuk and the other, anonymous, reviewer(s) for their contribution to the peer review of this work.

Reprints and permission information is available at http://www.nature.com/reprints

Publisher's note Springer Nature remains neutral with regard to jurisdictional claims in published maps and institutional affiliations.

Open Access This article is licensed under a Creative Commons Attribution 4.0 International License, which permits use, sharing, adaptation, distribution and reproduction in any medium or format, as long as you give appropriate credit to the original author(s) and the source, provide a link to the Creative Commons license, and indicate if changes were made. The images or other third party material in this article are included in the article's Creative Commons license, unless indicated otherwise in a credit line to the material. If material is not included in the article's Creative Commons license and your intended use is not permitted by statutory regulation or exceeds the permitted use, you will need to obtain permission directly from the copyright holder. To view a copy of this license, visit http://creativecommons.org/ licenses/by/4.0/.

(C) The Author(s) 2021 